\title{
Adult Stem Cells in Hibernation: Future Perspectives of Space Travel
}

\author{
Yu-Chih Chen ${ }^{1}$, Shin-Peir Aui ${ }^{2}$, Yin-Siew Lai ${ }^{2,3,4}$, Ko-Tung Chang ${ }^{2,3,4}$ \\ ${ }^{1}$ Graduate Institute of Bioresources, National Pingtung University of Science and Technology, Neipu, Taiwan \\ ${ }^{2}$ Department of Biological Science and Technology, National Pingtung University of Science and Technology, Neipu, Taiwan \\ ${ }^{3}$ Flow Cytometry Center, Precision Instruments Center, National Pingtung University of Science and Technology, Neipu, Taiwan \\ ${ }^{4}$ Research Center for Animal Biologics, National Pingtung University of Science and Technology, Neipu, Taiwan
}

Space traveling is imperative for mankind in the future. Expectedly, hibernation will become an option for space traveler to overcome the endless voyage. With regard to some of the studies pointed out that during hibernation, muscle will undergo atrophy and meantime neurogenesis will reduce, these obstacles were frequently related with stem cell regeneration. Thus, investigation on whether hibernation will lead to dysfunction of stem cell becomes an important issue. By going through four main systems in this article, such as, hematopoietic system, skeletal muscle system, central nervous system and orthopedic system, we are expecting that stem cells regeneration capacity will be affected by hibernation. To date, these researches are majorly the read-out from short term or seasonal hibernating mammals. Proposing and creating a simulated long-term hibernation animal model is turning essential for the further investigation on the effect of longer period of hibernation to human stem cells.

Keywords: Stem cell, Hibernation, Torpor, Arousal

\section{Introduction}

Imagine human who start setting out their journey to interstellar with the intent of exploring new planets. In March 2018, according to CEO from SpaceX, Elon Musk announced that a rocket to put humans on Mars could launch in early 2019 (1). By that time, when we are on

Received: April 8, 2019, Revised: June 20, 2019,

Accepted: July 5, 2019, Published online: August 31, 2019

Correspondence to Ko-Tung Chang

Department of Biological Science and Technology, National Pingtung University of Science and Technology, 1 Shuefu Rd., Neipu, Pingtung 91201, Taiwan

Tel: +886-8-7703202, Fax: +886-8-7740550

E-mail: kotungc@mail.npust.edu.tw

(c) This is an open-access article distributed under the terms of the Creative Commons Attribution Non-Commercial License (http://creativecommons.org/ licenses/by-nc/4.0/), which permits unrestricted non-commercial use, distribution, and reproduction in any medium, provided the original work is properly cited.

Copyright (c) 2019 by the Korean Society for Stem Cell Research board sailing out of The Solar System, we probably need to hibernate in a cramped hibernaculum in the spacecraft for years to decades to "slow down" our biological time. During hibernation period, we very likely will ask whether stem cells in our body continuously regenerate the damaged tissues? Or on the other hand, we may also question whether stem cells are still capable of proceeding self-renewal and differentiation when we are arousal from the endless journey? This is definitely not just a scenario from the recent movie "Passengers" in 2016; on the contrary, it is an interesting topic pending on more inspection. By this review article, we can understand more about the biology of stem cells that undergo hibernation. We wish to recruit more scientists together to dig out more findings on it and help mankind of us in the future to accomplish the mission of new earth exploration.

The term "hibernation" is commonly applied to all types of winter dormancy in vertebrate animals. As an adaptation to adverse winter conditions by certain mammals, hibernation is a state of greatly reduced metabolic 
activity and lowered body temperature. Hibernators include many fishes, amphibians, and reptiles that overwinter with body temperatures near freezing, as well as bears and a few other mammals that spend most of the winter sleeping in dens (2). Meanwhile, hibernation has been documented in eight different groups of mammals, such as monotremes, rodents, bats, insectivores, shrews, marsupials, primates (some lemurs), and carnivores (bears) (3). Preparation for hibernation begins in late summer when animals go through a period of hyperphagia by great increases of body fat (sometimes doubling body mass) and mainly stored as triglycerides in white adipose tissue (3). Hibernating mammals can tolerate extremes in temperature, oxygen saturation, organ perfusion, calorie intake, and immobilization (4). Besides, hibernation in mammals leads to profound reductions of metabolism, heart rate, blood flow, respiratory rate and core body temperature. These events do not represent a loss of homeostasis; instead, they are precisely controlled and spontaneously reversible (5). During hibernation, small mammals undergo long periods of deep torpor where their core body temperature can drop as low as $0 \sim 5^{\circ} \mathrm{C}$ (6). The low temperature of an animal in hibernation results in reduction of neural firing rates in the central nervous system (CNS) as well as cell metabolism that contributes to energy conservation for the animal to survive in winter when food is scarce (7). However, in the case of bears, their body temperature remains relatively stable during hibernation with a less dramatic drop in comparison to other hibernators (from $37^{\circ} \mathrm{C}$ in summer to $33 \sim 30^{\circ} \mathrm{C}$ in winter) (4).

Due to the well definitions of hibernation, researchers preferred these special hibernating mammals, for instances, 13-lined ground squirrels, Syrian hamsters and bears, as the models when carried out researches on hibernation issues. However, among all these hibernating mammals, small hibernating mammals will undergo torpor-arousal cycles that multiple bouts of deep torpor can last for days or weeks interspersed with periods of arousal, and by that time animals rewarm to the euthermic state for about a day (3). In this article by reviewing through the hematopoietic system, skeletal muscle system, central nervous system and orthopedic system from these hibernating mammals, researchers found out the influences of hibernation to the stem cells regeneration on these mammals. These systems play an important role in maintaining the life of the astronauts especially during the space voyage. Thus, the results from the hibernating mammals can be the references for us to face the same problems that might be appeared on the hibernating astronauts when they are experiencing such a long space journey.

\section{Study Analysis}

\section{Hematopoietic system}

Quiescence is a property that often characterizes tissue-resident stem cells and allows them to act as a dormant reserve that can replenish tissues during homeostasis (8). Quiescence is crucial for the self-renewal of adult hematopoietic stem cells (HSCs); furthermore, they must retain the capacity to proliferate rapidly, albeit transiently, in response to extrinsic cues that signals pathogenic infection or tissue injury (9). Previous study proved that most of the HSCs reside in the bone marrow (BM) niche while remaining in a state of quiescence, $G_{0}$ phase, and are recruited into the cell cycle at long intervals (10). The capacity to enter and to leave a hibernation-like state is one of the properties for HSCs to maintain 'stemness' and to prevent premature HSCs from exhaustion under conditions of hematopoietic stress $(11,12)$. This can be accorded by another research report that almost $75 \%$ of long-term (LT)-HSCs are quiescent in $\mathrm{G}_{0}$ at any moment, and $99 \%$ of LT-HSCs regularly divide every 57 days on average (13). In 2006, Yamazaki and others also proved that most $\mathrm{CD}^{-} 4^{-} \mathrm{c}_{\mathrm{kit}}{ }^{+} \mathrm{Sca}-{ }^{+} \mathrm{Lin}^{-}$(KSL) cells (HSC) that freshly isolated from mouse BM did not express Ki-67 and scarcely incorporated Pyronin Y, while most CD34 ${ }^{+}$ KSL progenitors did, suggesting that CD34- ${ }^{-} \mathrm{KSL}$ cells are in the $\mathrm{G}_{0}$ phase of the cell cycle (11). From evidence indicated that osteoblasts in the BM niche influence HSC cell cycle status. Arai et al. (14) found that quiescent HSCs adhere to osteoblastic cells of the BM through the interaction of the Tie2 receptor with its ligand angiopoietin-1 (Ang-1). Tight adhesion of HSCs to the osteoblastic niche leads to maintenance of quiescence. Furthermore, in another study showed that the thrombopoietin receptor $\mathrm{MPL}^{+}$HSCs closely contacted to thrombopoietin (THPO)producing osteoblastic cells at the endosteal surface in the trabecular bone area (15). Inhibition of THPO/MPL pathway by AMM2 (anti- MPL neutralizing antibody) results in a reduction of quiescence of LT-HSC, indicating that THPO/MPL signaling plays a critical role for LT-HSC regulation in the osteoblastic niche (15). Hematopoietic niche in BM consist of non-myelinating Schwann cells/glial cells glial fibrillary acidic protein (GFAP) that are ensheathed in autonomic nerves which play a role in regulating transforming growth factor beta (TGF- $\beta$ )- producing cells by processing latent TGF- $\beta$ into active TGF$\beta$. Meanwhile, activated latent TGF- $\beta$ will induce hibernation of hematopoietic stem cells (HSCs) to maintain it "stemness" which brought about higher percent of chimerism in mice; otherwise, with inactivated form of latent 
TGF- $\beta$ will negatively regulate the "stemness" of HSCs by inducing the cell cycle and resulting in loss of HSCs and low percent of chimerism (16). Another report showed that freshly isolated and cultured hibernating single HSCs exhibited long-term repopulating activity in the presence of TGF- $\beta$, while single HSCs cultured without TGF- $\beta$, they robustly proliferated but lost long-term repopulating activity (12). Besides, study unveiled that active TGF- $\beta$ triggered Smad signaling pathway at the same time efficiently inhibit cytokine-induced lipid raft clustering and elicited sustained accumulation of FoxO transcription factors and the induction of HSCs hibernation (10, 16). Moreover, study also reported that lipid rafts are diffusely distributed on freshly isolated CD $34^{-}$KSL HSCs but are highly polarized or clustered on $\mathrm{CD} 34^{+} \mathrm{KSL}$ progenitor cells. On the other hand, the activated TGF- $\beta$ receptor complex phosphorylates receptor-activated Smads (RSmad2) and R-Smad3 (12). Smad2 and Smad3 were activated in most HSCs, whereas only $25 \%$ of HSCs were in contact with the glial component of the niche (16). This finding raises the possibility that HSCs shift among various compartments of the niche depending on their activation status. Once Smad 2 and Smad 3 are activated via the glial component of the niche, HSCs may retain Smads in active form, even after relocation into another compartment, until the HSCs receive cues for cell division (16). On the contrary, while shifting away from glial cells, HSCs will able to form lipid raft clustering via downregulated Smad signaling. As a result, HSCs will be aroused and enter cell cycle (16). From above, we hypothesized that during space travel hibernation, glial cells might remain torpid and downregulate the TGF- $\beta$ -producing cells and activated latent TGF- $\beta$. As a result, the HSCs will recruit into cell cycle and expectedly loss their "stemness". Therefore, to prevent astronauts from suffering anemia after the endless space travel hibernation, it is important to investigate how to remain the function of the regulator glial cells intact.

\section{Skeletal muscle system}

Myostatin is a member of the TGF- $\beta$ superfamily and usually increased early in disuse and under hypocaloric conditions (17). When myostatin level increase, it will suppress satellite cell (postnatal muscle stem cells) to undergo differentiation into myofibres (5) and lead to muscle atrophy (17). However, studies proved in hibernation mammalian of 13-lined ground squirrel, myostatin level showed no significant increases during hibernation, but otherwise increased significantly during early arousal. At the same time, authors found that the increase of folli- statin levels by $\sim 1.5$-fold during hibernation and significant reduction in follistatin level during arousal (17). Follistatin is a myostatin-binding protein that can inhibit myostatin function and promote muscle growth (18). The expression of follistatin was also coincidence with a cell cycle inhibitor, p21, by a $224 \%$ increased in p21 protein during early torpor (ET) while significantly reduced by $68 \%$ during late torpor (LT) (6). The p21 protein, however, encourages satellite cells (SCs) differentiation. Due to above, SCs proliferation halted in deep torpor possibly in response to the earlier elevation in p21 (6).

Interestingly, Brooks also proved that SCs are not dormant during hibernation and showed the percentage of paired box protein $\left(\mathrm{Pax}^{+}\right)$SCs increased significantly during early torpor $(7.35 \pm 1.04 \%$ vs. control: $4.18 \pm 0.58 \%$; $\mathrm{p}<0.05)$ and returned to control levels during late torpor (LT) (6). The activated SCs that express both Pax7 and Myogenic Factor 5 (Myf5) will undergo self-renewal while SCs that express Pax7 only will undergo proliferation during early torpor (ET). Myf5 has been reported to play a particular role in promoting self-renewal of SCs and to facilitate the return of at least one self-cell to the pool of quiescent SCs $(6,19)$. Since protein level of Myf5 increased as well as protein level of p21 during ET, while insignificant change of Myf5 protein and drastically decrease of p21 protein level during LT, it implies that self-renewal of SCs still carry out during LT while proliferation and differentiation process was dramatically reduced. In addition, high level of helix-loop-helix muscle-specific transcription factor, MyoD, is involved in myogenic differentiation and SCs fusion with adult muscle fibers. Study proved that the murine $\mathrm{MyoD}^{-/-}$isolated satellite cells continue to proliferate and only a very small amount of cells transit into the myogenin-positive state, whereas the wild type cells exit the proliferative compartment and enter the myogenin-positive stage (20). Therefore, SCs expressing more MyoD than Myf5 confers for differentiation. Nonetheless, protein level of $\beta$-catenin was seen significantly increased during EA despite the lack of significant changes in Wnt4 (6). $\beta$-catenin is a key component of the canonical Wnt pathway $(21,22)$. $\beta$ catenin translocates into the nucleus where it binds to transcription factor called T-cell factor/lymphoid enhancer-binding factor (TCF/LEF) to activate transcription of Wnt-responsive genes involved in differentiation and cell proliferation (Cyclin D1, Myf5) (23).

Based on these evidences, it proved that SCs were not in dormant status during hibernation. Besides, proliferation and differentiation of SCs occur as well as self-renewal during hibernation. However, this phenomen- 
on happened on seasonal hibernating mammals' models that undergo torpid-arousal cycle. It definitely needs for further study on long-term hibernating model.

\section{Central nervous system}

Previous study demonstrated that decreased brain temperature shifts the major function of the hippocampus in the Syrian hamster (Mesocricetus auratus) from memory formation to increasing hibernation bout duration through suppression of ascending arousal system (7). The human ascending reticular activating system (ARAS) is an essential component of human consciousness mediating arousal (24). Through high angular resolution diffusion imaging (HARDI) tractography, the connectivity of ARAS was potentially being studied in its physiological manifestations, for example, sleep-wake cycling and anesthesia (24).

Adult neural stem cells generate new neurons in the dentate gyrus (DG) of the hippocampus and the lateral walls of the lateral ventricles (25). Adult neurogenesis is the most robust form of plasticity in the adult brain and likely contributes to memory formation (26). Besides that, Leó n-Espinosa and others elucidated that in hibernating Syrian hamster, a bunch of glial cells that express low brain lipid-binding protein (BLBP) in the dentate gyrus (DG) of the hippocampus and the subventricular zone (SVZ). At the same time, the low expression of the standard neurogenesis marker, such as phosphohistone-H3 (PHH3), doublecortin (DCX), and BLBP as well as the decreased incorporation of 5-bromo-2-deoxyuridine (BrdU), from the progenitor cells on DG and the neuroblasts or multipotent astrocytic-like cells on SVZ during hibernation demonstrated the decrease in cell cycle, neurogenesis and proliferation capacity. Simultaneously, tau3R, a DG adult neurogenesis marker, labeling on the neuronal axon in the SVZ and DG decreased in hibernating Syrian hamster, when compared with non-hibernating animals. Meanwhile, study showed clear reduction in the expression of polysialylate-neural cell adhesion molecule (PSA-NCAM), as an immature neurons marker, in the SVZ of torpid Syrian hamsters. Correspondingly, the number of PSANCAM labeled immature neuron cells in the DG is not diminished drastically but showing significant reduction of dendritic length and ramifications of DG cells from torpid hamsters (25). Since neurogenesis was reduced in hibernation state, the connectivity of ARAS between brainstem to thalamus, hypothalamus, hippocampal and basal forebrain would be impacted by the reduction of dendrite during hibernation. On the other hand, we predict that long lasting degeneration of ARAS connectivity in astronauts who undergo endless space journey during hiber- nation may cause incapability of returning to consciousness status even when their basal body temperature are increased to normal. As a result, the phenomenon may further cause them dying in coma and stay persistently in vegetative state. Thus, this certainly will be a crucial issue for deeper investigation.

\section{Orthopedic system}

Spaceflight, in particular microgravity, presents a unique set of physiological and environmental stresses. For all living organisms, including humans, microgravity exposure can lead to changes in muscle atrophy and bone loss (27). Despite many in vitro and in vivo studies under both simulated conditions and real microgravity, the mechanism of bone loss is still not very well-defined (27). In space, the lack of gravity or, rather, its extreme reduction, transforms the skeleton into a hypodynamic, which refers to reduction in the mechanical loading that occurs in all muscle of the body, and hypokinetic, referring to the reduction of movement model (28). Disuse typically causes an imbalance in bone formation and bone resorption that lead to trabecular bone loss. In humans, 12 weeks of bedrest increased bone resorption indices (eroded and osteoclast surfaces) by $100 \sim 250 \%$, but did not change indices of bone formation (osteoid and osteoblast surfaces) in the ilium (29). Disuse-induced bone loss is often associated with unbalanced bone remodeling (bone resorption and/or bone formation), which leads to increased concentrations of serum and urinary calcium (30-32). There are four major types of bone cells, based on their morphologies, functions, and locations: mesenchymal stem cells (MSCs), osteocytes, osteoblasts, and osteoclasts. MSCs have a spindle-shaped and a fibroblast-like morphology that possess capacity for self-renewal and multilineage differentiation potential into adipocytes, osteoblasts, and chondroblast. These properties are crucial for tissue repair after injury and are emerging as tools for regenerative medicine and tissue engineering (27,33). Interestingly, the report elucidated that human bone mesenchymal stem cell (hMSC) that culture in the microgravity condition created by using the Random Positioning Machine (RPM) induces the expression of protein level of several markers of osteogenesis, such as runt-related transcription factor 2 (RUNX2) and osteric (OSX) (33).

In contrast, research on hibernating black bear reported that bone remodeling is suppressed during hibernation with evidence illustrated that serum level bone formation marker, bone-specific alkaline phosphatase (BSALP), gradually reduced from pre-hibernation to hibernation and an increase from hibernation through the transition 
out of hibernation. The serum level of bone resorption marker tartrate-resistant acid phosphatase (TRACP) also showed significant changes, with a gradual decline from pre-hibernation to hibernation and a gradual increase during the transition out of hibernation, reaching its lowest value during hibernation. Similarly, histological analysis showed a reduced number of osteoblasts and osteoclasts on bone surfaces during hibernation compared with prehibernation. Because bone remodeling is metabolically expensive, increased bone remodeling, which typically occurs with disuse during hibernation would compete for energy stores with other physiological functions critical for survival, such as cardiac output and respiration (31). Study reported that under energy metabolism, both serum glucose and insulin were significantly lower during hibernation than in the pre-hibernation period (31).

According to report, mesenchymal stem cells and adipose-derived stem cells share many characteristics in common, such as the growth kinetics, antigen expression and multi-lineage differentiation capacity (34). Interestingly, in the den, hibernating brown bears do not develop tissue atrophy, despite almost no physical activity. Study proved by adipose-derived stem cells obtained from wild brown bears leaving the den $7 \sim 10$ days after the hibernating season. Those cells exposed to osteogenic growth factors cells spontaneously formed nodules that stained positive for Alizarin red, indicating calcium mineral deposition. While the cells cultured in chondrogenic induction media was stained extracellularly deposition of glycosaminoglycans with Alcian blue assessing chondrogenic differentiation. Moreover, the cells from three yearlings showed remarkable spontaneous cartilage and bone formation capacity and stem cell proliferation rate, while cells from 16-year-old bear showed negative staining with Alizarin red and Alcian blue plus very low rate of proliferation. This ability appears to be lost gradually with age (35).

Based on these data, further investigation is needed to explore that trilineage differentiation capacity whether it is linked to aging in hibernating animals. The role of MSCs in hibernating astronaut, especially for bone remodeling during space travel under microgravity condition would be addressed.

\section{Perspective}

According to the aforementioned, the effects of hibernation on stem cells were resulted from the seasonal hibernating mammals. Hence, the best and most accurate method is to send human into a hundred-year hibernation status for further researches. In other words, the major prem- ise by creating long-term hibernating mammals becomes certainly necessary. Unveil the specific regulatory protein that induce each states of torpor, for example early torpor, interbout torpor (deeper sleep) and late torpor by using next generation sequencing (NGS) analysis could be one of the approaches in the next place.

\section{Acknowledgments}

This study was supported by the Ministry of Science and Technology in Taiwan (grant MOST 106-2314-B020-001, MOST 108-3017-F-020-001 and MOST 108-2314B-020-002).

\section{Potential Conflict of Interest}

The authors have no conflicting financial interest.

\section{Author Contributions}

Chen Y.C., Aui S.P., Lai Y.S. and Chang K.T. conceived the study and drafted the manuscript; Chang K.T. approved the final version of the article.

\section{References}

1. Morris DZ. Elon Musk says SpaceX's Mars rocket could launch in early 2019 [Internet]. Fortune; 2018 Mar 11 [cited 2018 Jun 13]. Available from: http://fortune.com/2018/03/11/elon-musk-spacex-mars-rocket-bfrlaunch-timeline/.

2. The Editors of Encyclopaedia Britannica. Hibernation [Internet]. Chicago: Encyclopædia Britannica; 2018 Jan 5 [cited 2018 Jun 12]. Available from: https://www.britannica.com/science/hibernation.

3. Storey KB. Out cold: biochemical regulation of mammalian hibernation - a mini-review. Gerontology 2010;56:220-230

4. Sahdo B, Evans AL, Arnemo JM, Fröbert O, Särndahl E, Blanc S. Body temperature during hibernation is highly correlated with a decrease in circulating innate immune cells in the brown bear (Ursus arctos): a common feature among hibernators? Int J Med Sci 2013;10:508-514

5. Andres-Mateos E, Mejias R, Soleimani A, Lin BM, Burks TN, Marx R, Lin B, Zellars RC, Zhang Y, Huso DL, Marr TG, Leinwand LA, Merriman DK, Cohn RD. Impaired skeletal muscle regeneration in the absence of fibrosis during hibernation in 13-lined ground squirrels. PLoS One 2012;7:e48884

6. Brooks NE, Myburgh KH, Storey KB. Muscle satellite cells increase during hibernation in ground squirrels. Comp Biochem Physiol B Biochem Mol Biol 2015;189:55-61

7. Arant RJ, Goo MS, Gill PD, Nguyen Y, Watson KD, Hamilton JS, Horowitz JM, Horwitz BA. Decreasing temperature shifts hippocampal function from memory formation to modulation of hibernation bout duration in Syrian hamsters. Am J Physiol Regul Integr Comp Physiol 


\section{1;301:R438-R447}

8. Nakamura-Ishizu A, Takizawa H, Suda T. The analysis, roles and regulation of quiescence in hematopoietic stem cells. Development 2014;141:4656-4666

9. Pietras EM, Warr MR, Passegué E. Cell cycle regulation in hematopoietic stem cells. J Cell Biol 2011;195:709-720

10. Yamazaki S, Nakauchi H. Bone marrow Schwann cells induce hematopoietic stem cell hibernation. Int J Hematol 2014;99:695-698

11. Yamazaki S, Iwama A, Takayanagi S, Morita Y, Eto K, Ema H, Nakauchi H. Cytokine signals modulated via lipid rafts mimic niche signals and induce hibernation in hematopoietic stem cells. EMBO J 2006;25:3515-3523

12. Yamazaki S, Iwama A, Takayanagi S, Eto K, Ema H, Nakauchi H. TGF-beta as a candidate bone marrow niche signal to induce hematopoietic stem cell hibernation. Blood 2009;113:1250-1256

13. Cheshier SH, Morrison SJ, Liao X, Weissman IL. In vivo proliferation and cell cycle kinetics of long-term self-renewing hematopoietic stem cells. Proc Natl Acad Sci U S A 1999;96:3120-3125

14. Arai F, Hirao A, Ohmura M, Sato H, Matsuoka S, Takubo $\mathrm{K}$, Ito K, Koh GY, Suda T. Tie2/angiopoietin-1 signaling regulates hematopoietic stem cell quiescence in the bone marrow niche. Cell 2004;118:149-161

15. Yoshihara H, Arai F, Hosokawa K, Hagiwara T, Takubo K, Nakamura Y, Gomei Y, Iwasaki H, Matsuoka S, Miyamoto K, Miyazaki H, Takahashi T, Suda T. Thrombopoietin/MPL signaling regulates hematopoietic stem cell quiescence and interaction with the osteoblastic niche. Cell Stem Cell 2007;1:685-697

16. Yamazaki S, Ema H, Karlsson G, Yamaguchi T, Miyoshi H, Shioda S, Taketo MM, Karlsson S, Iwama A, Nakauchi H. Nonmyelinating Schwann cells maintain hematopoietic stem cell hibernation in the bone marrow niche. Cell 2011;147:1146-1158

17. Brooks NE, Myburgh KH, Storey KB. Myostatin levels in skeletal muscle of hibernating ground squirrels. J Exp Biol 2011;214(Pt 15):2522-2527

18. Lee SJ, Lee YS, Zimmers TA, Soleimani A, Matzuk MM, Tsuchida K, Cohn RD, Barton ER. Regulation of muscle mass by follistatin and activins. Mol Endocrinol 2010;24: 1998-2008

19. Beauchamp JR, Heslop L, Yu DS, Tajbakhsh S, Kelly RG, Wernig A, Buckingham ME, Partridge TA, Zammit PS. Expression of CD34 and Myf5 defines the majority of quiescent adult skeletal muscle satellite cells. J Cell Biol 2000;151:1221-1234

20. Yablonka-Reuveni Z, Rudnicki MA, Rivera AJ, Primig M, Anderson JE, Natanson P. The transition from proliferation to differentiation is delayed in satellite cells from mice lacking MyoD. Dev Biol 1999;210:440-455

21. Otto A, Schmidt C, Luke G, Allen S, Valasek P, Muntoni F, Lawrence-Watt D, Patel K. Canonical Wnt signalling induces satellite-cell proliferation during adult skeletal muscle regeneration. J Cell Sci 2008;121(Pt 17):2939-2950
22. Bernardi H, Gay S, Fedon Y, Vernus B, Bonnieu A, Bacou F. Wnt4 activates the canonical $\beta$-catenin pathway and regulates negatively myostatin: functional implication in myogenesis. Am J Physiol Cell Physiol 2011;300:C1122C1138

23. Abiola M, Favier M, Christodoulou-Vafeiadou E, Pichard AL, Martelly I, Guillet-Deniau I. Activation of Wnt/beta-catenin signaling increases insulin sensitivity through a reciprocal regulation of Wnt10b and SREBP-1c in skeletal muscle cells. PLoS One 2009;4:e8509

24. Edlow BL, Takahashi E, Wu O, Benner T, Dai G, Bu L, Grant PE, Greer DM, Greenberg SM, Kinney HC, Folkerth RD. Neuroanatomic connectivity of the human ascending arousal system critical to consciousness and its disorders. J Neuropathol Exp Neurol 2012;71:531-546

25. León-Espinosa G, García E, Gómez-Pinedo U, Hernández F, DeFelipe J, Ávila J. Decreased adult neurogenesis in hibernating Syrian hamster. Neuroscience 2016;333:181-192

26. Gonçalves JT, Schafer ST, Gage FH. Adult neurogenesis in the hippocampus: from stem cells to behavior. Cell 2016; 167:897-914

27. Arfat Y, Xiao WZ, Iftikhar S, Zhao F, Li DJ, Sun YL, Zhang G, Shang P, Qian AR. Physiological effects of microgravity on bone cells. Calcif Tissue Int 2014;94:569-579

28. Vico L, Alexandre C. Microgravity and bone adaptation at the tissue level. J Bone Miner Res 1992;7 Suppl 2:S445S447

29. McGee-Lawrence ME, Wojda SJ, Barlow LN, Drummer TD, Castillo AB, Kennedy O, Condon KW, Auger J, Black HL, Nelson OL, Robbins CT, Donahue SW. Grizzly bears (Ursus arctos horribilis) and black bears (Ursus americanus) prevent trabecular bone loss during disuse (hibernation). Bone 2009;45:1186-1191

30. McGee-Lawrence ME, Wojda SJ, Barlow LN, Drummer TD, Bunnell K, Auger J, Black HL, Donahue SW. Six months of disuse during hibernation does not increase intracortical porosity or decrease cortical bone geometry, strength, or mineralization in black bear (Ursus americanus) femurs. J Biomech 2009;42:1378-1383

31. McGee-Lawrence M, Buckendahl P, Carpenter C, Henriksen $\mathrm{K}$, Vaughan M, Donahue S. Suppressed bone remodeling in black bears conserves energy and bone mass during hibernation. J Exp Biol 2015;218(Pt 13):2067-2074

32. Watanabe Y, Ohshima H, Mizuno K, Sekiguchi C, Fukunaga M, Kohri K, Rittweger J, Felsenberg D, Matsumoto T, Nakamura T. Intravenous pamidronate prevents femoral bone loss and renal stone formation during 90-day bed rest. J Bone Miner Res 2004;19:1771-1778

33. Cazzaniga A, Maier JAM, Castiglioni S. Impact of simulated microgravity on human bone stem cells: new hints for space medicine. Biochem Biophys Res Commun 2016;473: 181-186

34. Zhang DZ, Gai LY, Liu HW. [Differences between adipose-derived stem cells and mesenchymal stem cells in differentiation into cardiomyocytes]. Sheng Li Xue Bao 2008; 60:341-347. Chinese 
35. Fink T, Rasmussen JG, Emmersen J, Pilgaard L, Fahlman Å, Brunberg S, Josefsson J, Arnemo JM, Zachar V, Swenson JE, Fröbert O. Adipose-derived stem cells from the brown bear (Ursus arctos) spontaneously undergo chondrogenic and osteogenic differentiation in vitro. Stem Cell Res 2011; 7:89-95 